PROCEEDINGS OF THE

AMERICAN MATHEMATICAL SOCIETY

Volume 128, Number 9, Pages 2603-2609

S 0002-9939(00)05329-6

Article electronically published on March 1, 2000

\title{
TOPOLOGICAL ENTROPY, EMBEDDINGS AND UNITARIES IN NUCLEAR QUASIDIAGONAL $C^{*}$-ALGEBRAS
}

\author{
NATHANIAL P. BROWN
}

(Communicated by David R. Larson)

\begin{abstract}
Using topological entropy of automorphisms of $C^{*}$-algebras, it is shown that some important facts from the theory of AF algebras do not carry over to the class of $A \mathbb{T}$ algebras.

It is shown that in general one cannot perturb a basic building block into a larger one which almost contains it. The same entropy obstruction used to prove this fact also provides a new obstruction to the known fact that two injective homomorphisms from a building block into an $A \mathbb{T}$ algebra need not differ by an (inner) automorphism when they agree on K-theory.
\end{abstract}

\section{INTRODUCTION}

The approximation approach to topological entropy was first conceived by Voiculescu (see [Vo2 for the nuclear case or Br2] for the exact case). The purpose of this note is to show how certain geometric results can be easily derived from some basic properties of the topological entropy function $h t$.

Recall that a $C^{*}$-algebra $A$ is called $A \mathbb{T}$ if it is isomorphic to an inductive limit of circle algebras (direct sums of matrix algebras over $C(\mathbb{T})$ ). It follows that if $A$ is $A \mathbb{T}$, then one can write $A=\bar{\bigcup} A_{i}$, where $A_{i} \subset A_{i+1}$ and each $A_{i}$ is a "basic building block" of the form $M_{n_{1}}\left(C\left(X_{1}\right)\right) \oplus \cdots \oplus M_{n_{k}}\left(C\left(X_{k}\right)\right)$, where each $X_{j}$ is homeomorphic to $\mathbb{T},[0,1]$ or a point $([\mathrm{El}])$. Now consider the following questions concerning $A \mathbb{T}$ algebras.

Question 1.1. If $A$ is $A \mathbb{T}$, and $B$ and $C$ are subalgebras with $B$ isomorphic to a building block and nearly contained in $C$, then does there exist a unitary $u \in A$ such that $A d u(B) \subset C$ ? More generally, is it possible to write $A=\overline{\bigcup A_{i}}$, where each $A_{i} \subset A_{i+1}$ is a building block and $B=A_{1}$ ?

Question 1.2. If $B$ is a basic building block and $\varphi, \psi: B \rightarrow A$ are two *monomorphisms such that $\varphi_{*}=\psi_{*}: K_{*}(B) \rightarrow K_{*}(A)$, then is it possible to find a unitary $u \in A$ such that $A d u \circ \varphi=\psi$ ? Or even just an automorphism $\alpha \in A u t(A)$ such that $\alpha \circ \varphi(B) \subset \psi(B)$ ?

Received by the editors October 14, 1998.

1991 Mathematics Subject Classification. Primary 46L05, 46L80, 46L55.

Key words and phrases. Topological entropy, (generalized) inductive limits, inner automorphisms, embeddings.

This work was partially supported by an NSF Dissertation Enhancement Award.

(C)2000 American Mathematical Society 
Note that in the theory of AF algebras the finite dimensional analogues of these questions have affirmative answers and play crucial roles in understanding AF algebras. The first yields a local characterization of AF algebras and the second a K-theoretic classification (cf. [Da]).

It is known that there is a spectral multiplicity obstruction to conjugating unitaries with full spectrum in UHF algebras and hence the answer to the first part of Question 1.2 is known to be negative. We will show that entropy provides another obstruction which also yields negative answers to Question 1.1 and the second part of Question 1.2 In particular, this illustrates just how delicate Elliott's local characterization of $A \mathbb{T}$ algebras is and that his approximately intertwining diagram technique is not a tool of convenience but rather of necessity (see [E] ).

Our strategy is straightforward. We simply show that there is a dynamical obstruction to constructing an increasing nest of building blocks (with dense union) over certain unitaries in $A \mathbb{T}$ algebras. It turns out that this obstruction does not disappear by passing to larger algebras and hence we will show that there exist (many) unitaries $u$ in the Universal UHF algebra, $\mathcal{U}=\bigotimes_{n=1}^{\infty} M_{n}(\mathbb{C})$, with the following property:

(*) If $\rho: \mathcal{U} \rightarrow A$ is an embedding into an $A \mathbb{T}$ algebra $A$, then it is impossible to write $A=\bar{\bigcup} A_{i}$ where each $A_{i} \subset A_{i+1}$ is a building block and $\rho(u) \in A_{1}$.

Geometrically it is not clear (at least to this author) what prevents a given unitary from being embedded into an increasing nest of building blocks of a larger algebra, but dynamically it will become very clear.

Section 2 of this note contains the main technical result. Namely, that the topological entropy of inner automorphisms of subhomogeneous algebras is always zero. Then in section 3 we discuss some consequences of this result, including the resolution of the questions above.

\section{INNER AUTOMORPHISMS OF SUBHOMOGENEOUS ALGEBRAS}

We refer the reader to $\mathrm{Br} 2$ for the definition of the topological entropy function $h t$ as well as all the related notation which appears below (see also [Vo2]).

The following lemma will be the key to proving that inner automorphisms of subhomogeneous algebras have zero entropy.

Lemma 2.1. Let $A=M_{m}(C(X))=C\left(X, M_{m}\right)$ be a homogeneous $C^{*}$-algebra (with $X$ a compact metric space), let $\omega=\left\{f_{1}, \ldots, f_{k}\right\} \subset A$ with $\left\|f_{i}\right\| \leq 1$ for $i=1, \ldots, k$, and let $\delta>0$ be given. If $\left\{\gamma_{n}\right\}$ is a sequence of real numbers with $\gamma_{n} \rightarrow \infty$, then there exists a sequence of positive integers $\left\{K_{n}\right\}$ and a constant $C=C(k, \delta, m)>0$ which is independent of $n$ and maps

$$
\begin{gathered}
\varphi_{n}: A \rightarrow \bigoplus_{1}^{K_{n}} M_{m}(\mathbb{C}), \\
\psi_{n}: \bigoplus_{1}^{K_{n}} M_{m}(\mathbb{C}) \rightarrow A
\end{gathered}
$$

such that $\varphi_{n}$ are point evaluations (and hence ${ }^{*}$-homomorphisms), $\psi_{n}$ are unital completely positive maps and

1) $\left\|\psi_{n} \circ \varphi_{n}\left(f_{i}\right)-f_{i}\right\| \leq \delta / \gamma_{n}$ for $i=1, \ldots, k$,

2) $K_{n} \leq C \gamma_{n}^{C}$ for all $n \in \mathbb{N}$. 
Proof. First we identify $M_{m}(\mathbb{C})$ with $\mathbb{R}^{2 m^{2}}$ and let $\Omega \subset \mathbb{R}^{2 m^{2}}$ be a cube containing the unit ball of $M_{m}(\mathbb{C})$ (and hence the ranges of all the $f_{i}$, since $\left\|f_{i}\right\| \leq 1$ ). Now construct a sequence of open covers of $\Omega$, say $\Omega_{n}=\left\{\Omega_{1}^{(n)}, \ldots, \Omega_{\tilde{K}_{n}}^{(n)}\right\}$, such that $\Omega_{i}^{(n)}$ is a cube of diameter $<\delta /\left(\lambda \gamma_{n}\right)$ for $i=1, \ldots, \tilde{K}_{n}$, where $\lambda$ is a constant giving the equivalence of norms in $M_{m}(\mathbb{C})$ and $\mathbb{R}^{2 m^{2}}$. It is not hard to verify that this can be done in such a way that the number of cubes (i.e. $\tilde{K}_{n}$ ) is bounded above by $\left(C_{1} \gamma_{n}\right)^{2 m^{2}}$, where $C_{1}$ is a constant depending only on $\delta$ and $m$ (note that the diameter of $\Omega$ depends on $\lambda$ which in turn only depends on $m$ ). Note that transferring each $\Omega_{i}^{(n)}$ back to $M_{m}(\mathbb{C})$, we have open sets with diameter $<\delta / \gamma_{n}$.

Now we construct (for each $n$ ) a family of open covers of $X$ which are just the pullbacks of $\Omega_{n}$ through each of the functions $f_{i}$. That is, we let

$$
\mathcal{O}_{f_{i}}^{(n)}=\left\{U_{f_{i}, j}^{(n)}\right\}_{1 \leq j \leq \tilde{K_{n}}}, \text { where } U_{f_{i}, j}^{(n)}=f_{i}^{-1}\left(\Omega_{j}^{(n)}\right), \quad 1 \leq j \leq \tilde{K}_{n} .
$$

Finally, we take the join of $\mathcal{O}_{f_{1}}^{(n)}, \ldots, \mathcal{O}_{f_{k}}^{(n)}$ and denote it by $\mathcal{O}^{(n)}$. Thus

$$
\mathcal{O}^{(n)}=\mathcal{O}_{f_{1}}^{(n)} \vee \ldots \vee \mathcal{O}_{f_{k}}^{(n)}=\left\{V_{1} \cap \ldots \cap V_{k}: V_{j} \in O_{f_{j}}^{(n)}\right\} .
$$

Now write $\mathcal{O}^{(n)}=\left\{U_{j}^{(n)}\right\}_{1 \leq j \leq K_{n}}$ and note that by construction we have the following:

a) $K_{n} \leq\left(\tilde{K}_{n}\right)^{k} \leq\left(C_{1} \gamma_{n}\right)^{2 m^{2} k}$

b) if $x, y \in U_{j}^{(n)}$, then $\left\|f_{i}(x)-f_{i}(y)\right\| \leq \delta / \gamma_{n}$ for $1 \leq j \leq K_{n}$ and $1 \leq i \leq k$.

Letting our constant in the statement of the lemma be $C=\left(C_{1}\right)^{2 m^{2} k}$, we must now produce the maps $\varphi_{n}$ and $\psi_{n}$ to complete the proof. However, this is now a simple partition of unity argument.

So, choose points $x_{j}^{(n)} \in U_{j}^{(n)}$ and let $\left\{\Lambda_{j}^{(n)}\right\}_{1 \leq j \leq K_{n}}$ be a partition of unity subordinate to $\mathcal{O}^{(n)}$. Then we define

$$
\varphi_{n}: A \rightarrow \bigoplus_{1}^{K_{n}} M_{m}(\mathbb{C}) \text { by } f \mapsto f\left(x_{1}^{(n)}\right) \oplus \ldots \oplus f\left(x_{K_{n}}^{(n)}\right)
$$

and

$$
\psi_{n}: \bigoplus_{1}^{K_{n}} M_{m}(\mathbb{C}) \rightarrow A \text { by } T_{1} \oplus \ldots \oplus T_{K_{n}} \mapsto \sum_{j=1}^{K_{n}} T_{j} \Lambda_{j}^{(n)} .
$$

It is now routine to verify that $\varphi_{n}$ and $\psi_{n}$ are ${ }^{*}$-homomorphisms and completely positive maps, respectively, and that $\left\|\psi_{n} \circ \varphi_{n}\left(f_{i}\right)-f_{i}\right\| \leq \delta / \gamma_{n}$ for $i=1, \ldots, k$.

Lemma 2.2. Let $A=M_{m}(C(X))$ be a homogeneous $C^{*}$-algebra (with $X$ a compact metric space), and let $u \in A$ be a unitary. Then $h t(A d u)=0$.

Proof. Assume $A$ is faithfully represented on a Hilbert space $H$. Note that by the Kolmogorov-Sinai type result (cf. [Vo2, Prop. 4.3] or [Br2, Prop. 2.6]), it suffices to prove that $h t\left(i d_{A}, A d u, \omega\right)=0$ for all $\omega=\left\{f_{1}, \ldots, f_{k}\right\}$ with $\left\|f_{i}\right\| \leq 1$ as in the previous lemma. So let $\delta>0$ be given and let $\gamma_{n}=2 n+1$. Now applying the previous lemma to $\omega \cup\left\{u, u^{*}\right\}, \delta$ and $\left\{\gamma_{n}\right\}$ we can construct sequences

$$
\varphi_{n}: A \rightarrow \bigoplus_{1}^{K_{n}} M_{m}(\mathbb{C}), \quad \text { where } f \mapsto f\left(x_{1}^{(n)}\right) \oplus \ldots \oplus f\left(x_{K_{n}}^{(n)}\right),
$$


and

$$
\psi_{n}: \bigoplus_{1}^{K_{n}} M_{m}(\mathbb{C}) \rightarrow A, \quad \text { where } \quad T_{1} \oplus \ldots \oplus T_{K_{n}} \mapsto \sum_{j=1}^{K_{n}} T_{j} \Lambda_{j}^{(n)},
$$

where (as constructed in the previous lemma) $\left\{\Lambda_{j}\right\}_{1 \leq j \leq K_{n}}$ is a partition of unity subordinate to an open cover $\mathcal{O}^{(n)}=\left\{U_{j}^{(n)}\right\}_{1 \leq j \leq K_{n}}, x_{j}^{(n)} \in U_{j}^{(n)}$ for $1 \leq j \leq K_{n}$ and the $U_{j}^{(n)}$ also have the property that $x, y \in U_{j}^{(n)} \Rightarrow\left\|f_{i}(x)-f_{i}(y)\right\|<\delta / \gamma_{n}$ for $i=1, \ldots, k$ and all $n \in \mathbb{N}$.

Letting $B_{n}=\bigoplus_{1}^{K_{n}} M_{m}(\mathbb{C})$, we have that $\left(\varphi_{n}, \psi_{n}, B_{n}\right) \in C P A\left(i d_{A}, A\right)$ and

$$
\operatorname{rank}\left(B_{n}\right)=m K_{n} \leq m C \gamma_{n}^{C}=m C(2 n+1)^{C}
$$

(recall that $C$ is independent of $n$ ). Now we observe that for all $x \in U_{j}^{(n)}$ we have:

$$
\left\|u^{l}(x) f_{i}(x) u^{* l}(x)-u^{l}\left(x_{j}^{(n)}\right) f_{i}\left(x_{j}^{(n)}\right) u^{* l}\left(x_{j}^{(n)}\right)\right\| \leq(2 l+1)\left(\delta / \gamma_{n}\right) \leq \delta
$$

for $1 \leq i \leq k$ and $1 \leq l \leq n$. Hence

$$
\begin{aligned}
\left\|\psi_{n} \varphi_{n}\left(u^{l} f_{i} u^{* l}\right)-u^{l} f_{i} u^{* l}\right\| & =\left\|\sum_{j=1}^{K_{n}} u^{l}\left(x_{j}^{(n)}\right) f_{i}\left(x_{j}^{(n)}\right) u^{* l}\left(x_{j}^{(n)}\right) \Lambda_{j}^{(n)}-\sum_{j=1}^{K_{n}} u^{l} f_{i} u^{* l} \Lambda_{j}^{(n)}\right\| \\
& =\left\|\sum_{j=1}^{K_{n}}\left(u^{l}\left(x_{j}^{(n)}\right) f_{i}\left(x_{j}^{(n)}\right) u^{* l}\left(x_{j}^{(n)}\right)-u^{l} f_{i} u^{* l}\right) \Lambda_{j}^{(n)}\right\| \\
& \leq \delta .
\end{aligned}
$$

Thus we see that $r c p\left(i d_{A}, \omega \cup \ldots \cup A d u^{n}(\omega), \delta\right) \leq \operatorname{rank}\left(B_{n}\right) \leq m C(2 n+1)^{C}$ and hence

$$
h t\left(i d_{A}, A d u, \omega, \delta\right) \leq \limsup _{n \rightarrow \infty} \frac{\log \left(m C(2 n+1)^{C}\right)}{n+1}=0 .
$$

This implies that $h t\left(i d_{A}, A d u, \omega\right)=0$.

To get the same conclusion for inner automorphisms of subhomogeneous algebras we recall the following two results from $[\mathrm{Br} 2$.

Proposition 2.3 (cf. Prop. 2.9 in [Br2]). If $A=A_{1} \oplus \cdots \oplus A_{k}$ and $\alpha=\alpha_{1} \oplus \cdots \oplus$ $\alpha_{k} \in \operatorname{Aut}(A)$, then $h t(\alpha)=\max \left\{h t\left(\alpha_{1}\right), \ldots, h t\left(\alpha_{k}\right)\right\}$.

Proposition 2.4 (cf. Prop. 2.1 in $[\mathrm{Br} 2]$ ). (Monotonicity) If $A \subset B$ is a subalgebra such that $\alpha(A)=A$, where $\alpha \in \operatorname{Aut}(B)$, then $h t\left(\left.\alpha\right|_{A}\right) \leq h t(\alpha)$.

Corollary 2.5. If $A$ is isomorphic to a unital subalgebra of $M_{n_{1}}\left(X_{1}\right) \oplus \cdots \oplus$ $M_{n_{k}}\left(X_{k}\right)$, where the $X_{i}$ are compact metric spaces and $u \in A$ is a unitary, then $h t(A d u)=0$.

\section{EMBEDDINGS AND UNITARIES}

We will now give an alternative definition of the (unital) strong NF algebras introduced in [BK1]. For the record, by a subhomogeneous algebra we will always mean a unital subalgebra of $M_{n_{1}}\left(X_{1}\right) \oplus \cdots \oplus M_{n_{k}}\left(X_{k}\right)$, where the $X_{i}$ 's are compact metric spaces.

Definition 3.1. We will call a separable unital $C^{*}$-algebra $A$ a generalized $A S H$ algebra if there exists a generalized inductive system $\left(A_{i}, \Phi_{i, j}\right)$, in the sense of [BK1], with $A=\lim _{\longrightarrow}\left(A_{i}, \Phi_{i, j}\right)$, where each $A_{i}$ is a subhomogeneous algebra and the connecting maps $\overrightarrow{\Phi_{i, j}}$ are unital complete order embeddings. 
It is clear that a unital strong NF algebra is a generalized ASH algebra, while a generalized ASH algebra is strong NF by Corollary 5.1 of BK2 together with Corollary 6.1 .3 of [BK1]. Of course, the point is that we want to be able to use infinite dimensional building blocks when presenting the algebra.

Assume now that $A$ is a generalized ASH algebra and that $u \in A$ is a unitary. Assume further that we can write $A=\underline{\varliminf}\left(A_{i}, \Phi_{i}\right)$, where each $A_{i}$ is subhomogeneous and $u \in \Phi_{1}\left(A_{1}\right)$ (here, $\Phi_{i}: A_{i} \rightarrow A$ denotes the induced unital complete order embedding of $A_{i}$ into $A$ ). Then $A d u$ defines an inner automorphism of each $A_{i}$, which we will denote by $\left.A d u\right|_{A_{i}}$ (see Proposition 2.15 in [Br2] for the details). The following result is a special case of Proposition 2.15 in [Br2].

Proposition 3.2. In the situation above we have the following formula:

$$
h t(A d u)=\sup _{i} h t\left(\left.A d u\right|_{A_{i}}\right) .
$$

Note that every unital separable nuclear quasidiagonal $C^{*}$-algebra has a unital embedding into a generalized ASH algebra since every such algebra unitally embeds into a strong NF algebra (Cor. 5.8 of [BK2]). Also, since the strong NF algebras are closed under tensor products (Prop. 5.17 in [BK2]), it is worth pointing out that every separable nuclear quasidiagonal $C^{*}$-algebra has an embedding into a generalized ASH algebra where the building blocks are infinite dimensional.

Corollary 3.3. Let $A$ be a unital separable $C^{*}$-algebra, and let $u \in A$ be a unitary. If $h t(A d u)>0$, then there does not exist a unital ${ }^{*}$-monomorphism $\rho: A \rightarrow B$, where $B=\lim _{\longrightarrow}\left(B_{i}, \Phi_{i}\right)$ is a generalized $A S H$ algebra and such that $\rho(u) \in \Phi_{i}\left(A_{i}\right)$ for some $i \in \overrightarrow{\mathbb{N}}$.

Proof. The contrapositive of this statement follows easily from monotonicity of the entropy function (Proposition 2.4), Corollary 2.5 and Proposition 3.2 above.

Remark 3.4. In particular, this corollary implies that positive topological entropy of an inner automorphism is an obstruction to building an increasing nest of (sub)homogeneous algebras over a given unitary.

Counterexample to Questions 1.1 and 1.2. Let $M_{2 \infty}=\bigotimes_{-\infty}^{\infty} M_{2}(\mathbb{C})$ be the CAR algebra and $\alpha \in \operatorname{Aut}\left(M_{2 \infty}\right)$ the noncommutative Bernoulli shift (mapping each copy of $M_{2}$ one space to the right). Then by Proposition 4.7 in [Vo2] we have that $h t(\alpha)=\log 2$. Let $A=M_{2^{\infty}} \times_{\alpha} \mathbb{Z}$, and let $u \in A$ be the implementing unitary. From monotonicity we get that $h t(A d u)>0$. It was first shown in BKRS] (and later generalized in [Ki1 Ki2]) that $A$ is an $A \mathbb{T}$ algebra and hence Corollary 3.3 above implies that $u$ provides a counterexample to Question 1.1.

To prove that $u$ provides a counterexample to Question 1.2 we first note that $[u]$ is a nontrivial element of $K_{1}(A)([\mathrm{PV}])$. Since any unitary which is close to $u$ will give the same element in K-theory, we see that if $w$ is a unitary which lives in a dense nest of building blocks and is close to $u$, then $[w]$ is a nontrivial element of $K_{1}(A)$. In particular we have that the spectrum of $w$ is all of $\mathbb{T}$. But then the canonical maps from $C(\mathbb{T})$ into $A$ mapping the generator of $C(\mathbb{T})$ to $u$ and $w$, respectively, will both be injective and agree on K-theory. However, there can be no automorphism of $A$ which takes $u$ into $C^{*}(w)$, since the inverse of this automorphism would map a dense nest of building blocks onto $u$. 
To prove the existence of unitaries in the Universal UHF algebra $\mathcal{U}$ with the property $(*)$ of the introduction we simply note that $\mathcal{U}$ contains copies of every crossed product by $\mathbb{Z}$ of every other UHF algebra (cf. Thm. 3.6 in [Vo1]) and thus, in particular, has (many) unitaries which induce inner automorphisms with positive topological entropy.

Remark 3.5. It is thus no accident that in all the cases where crossed products by $\mathbb{Z}$ are known to be $A \mathbb{T}$ algebras the proofs have always approximated the implementing unitary by circle algebras rather than actually containing it (for example $\mathrm{Pu}$, Ki1, Ki2]). (It is interesting to note that this is also the case for the irrational rotation algebra, even though there is no entropy obstruction in this case; cf. [EE].)

It may turn out that many interesting crossed products of abelian algebras by minimal homeomorphisms can be realized as inductive limits of subhomogeneous algebras. (See $[\mathrm{LP}$ for some progress in this direction.) However, we conclude from our observations that in any attempt to decompose a crossed product as a limit of subhomogeneous algebras one must be sure not to include the implementing unitary in the decomposition (at least in the presence of positive entropy).

\section{ACKNOWLEDGEMENTS}

The author would like to thank his advisor, Professor Marius Dadarlat, for all of the time and support he has provided. He would also like to thank Professor Chris Phillips for several helpful conversations. Finally, he wishes to thank the NSF and the University of Tokyo for the opportunity to study in such an active environment.

\section{REFERENCES}

[BK1] B. Blackadar and E. Kirchberg, Generalized inductive limits of finite dimensional $C^{*}$ algebras, Math. Ann. 307 (1997), 343 - 380. MR 98c:46112

[BK2] B. Blackadar and E. Kirchberg, Inner quasidiagonality and strong NF algebras, Preprint.

[BKRS] O. Bratteli, A. Kishimoto, M. Rørdam and E. Størmer, The crossed product of a UHF algebra by a shift, Ergod. Th. Dynam. Sys. 13 (1993), 615 - 626. MR 95c:46111

[Br1] N.P. Brown, AF embeddability of crossed products of AF algebras by the integers, J. Funct. Anal. 160 (1998), 150-175. CMP 99:06

[Br2] N.P. Brown, Topological Entropy in Exact $C^{*}$-algebras, Preprint 1998.

[Da] K.R. Davidson, $C^{*}$-algebras by Example, Fields Inst. Monographs vol. 6, Amer. Math. Soc., (1996). MR 97i:46095

[El] G.A. Elliott, On the classification of $C^{*}$-algebras of real rank zero, J. reine angew. Math. 443 (1993), 179-219. MR 94i:46074

[EE] G.A. Elliott and D.E. Evans, The structure of the irrational rotation $C^{*}$-algebra, Ann. Math. 138 (1993), 477-501. MR 94j:46066

[Ki1] A. Kishimoto, The Rohlin property for automorphisms of UHF algebras, J. reine angew. Math. 465 (1995), 183 - 196. MR 96k:46114

[Ki2] A. Kishimoto, Unbounded derivations in AT algebras, J. Funct. Anal. 160 (1998), 270311. CMP 99:06

[LP] Q. Lin and N.C. Phillips, Ordered K-theory for $C^{*}$-algebras of minimal homeomorphisms, Advances in Operator Algebras and Operator Theory, Contem. Math. 228 (1998), 289314.

[Pi] M. Pimsner, Embedding some transformation group $C^{*}$-algebras into AF algebras, Ergod. Th. Dynam. Sys. 3 (1983), 613 - 626. MR 86d:46054

[PV] M. Pimsner and D. Voiculescu, Exact sequences for K-groups and Ext-groups of certain crossed products of $C^{*}$ - algebras, J. Oper. Th. 4 (1980), 93 - 118. [MR 82c:46074

$[\mathrm{Pu}] \quad$ I. Putnam, On the topological stable rank of certain transformation group $C^{*}$-algebras, Ergod. Th. and Dyn. Sys. 10 (1990), 197 - 207. MR 91f:46090 
[Vo1] D. Voiculescu, Almost inductive limit automorphisms and embeddings into AF algebras, Ergod. Th. Dynam. Sys. 6 (1986), 475 - 484. MR 88k:46073

[Vo2] D. Voiculescu, Dynamical approximation entropies and topological entropy in operator algebras, Comm. Math. Phys. 170 (1995), 249 - 281. MR 97b:46082

Department of Mathematics, Purdue University, West Lafayette, Indiana 47907-1901

E-mail address: nbrown@math.purdue.edu

Current address: Department of Mathematics, University of California-Berkeley, Berkeley, California 94720 